\title{
Seismic response and simulations of reinforced concrete bridge using OpenSees on high performance computing
}

\author{
Pallavi Gavali $\cdot$ Mahesh S. Shah · Gouri Kadam • \\ Kranti Meher
}

Received: 30 October 2012/Accepted: 14 May 2013/Published online: 2 August 2013

(C) CSI Publications 2013

\begin{abstract}
Nonlinear seismic analysis is becoming increasingly significant to grasp the performance of structures under earthquake. A nonlinear finite element model of existing bridge at Karad, India, including the bridge structure, pile groups, and the supporting foundation soil, is developed under 2D and 3D conditions in Gid (a pre and postprocessor software). The computational model is analyzed using Parallel OpenSees. OpenSees is open source software for carrying out earthquake engineering simulations, developed by Pacific Earthquake Engineering Research Centre, USA. The earthquake simulations were carried out using C-DAC's high performance computing facilities. The ground motion selection and modification technique-predicting median interstory drift response of building, ground motions are selected by $M$ and $R$ and scaling to $S a(T 1)$, is used for seismic response of combined large scale soil-structure interaction of Karad bridge. The idealized model properly represents the actual geometry; boundary conditions, gravity loads and mass distribution. Nonlinear modeling and analysis allows more accurate determination of stresses, strains, deformations and forces of critical components. The present work involves the effects of specially varying input excitation (earthquakes)
\end{abstract}

\author{
P. Gavali $(\varangle) \cdot$ M. S. Shah · G. Kadam · K. Meher \\ Computer Aided Engineering Group, Centre for Development of \\ Advanced Computing, Ganeshkhind, Pune, India \\ e-mail: pallavig@cdac.in \\ M. S. Shah \\ e-mail: mahesh@cdac.in \\ G. Kadam \\ e-mail: gourik@cdac.in \\ K. Meher \\ e-mail: krantim@cdac.in
}

at an existing bridge site. A nonlinear finite element model of this bridge site including the bridge structure, pile group and supporting foundation soil is developed in $2 \mathrm{D}$ plane strain conditions and in 3D 20 noded brick element. Carefully calibrated nonlinear stress-strain models are employed for both bridge and soil materials, in order to realistically reproduce actual site conditions. Seismic input motions are defined as forces using the boundary layer force method (zero length element approach). The earthquake simulation of bridge structure includes large scale interaction between structure-foundation-soil system and deformations at various locations of the bridge. The results include deformations at base of piers and at various spans of the bridge. Performing the bridge simulation on C-DAC's Param Yuva facility results in accuracy and saving in computing efforts.

Keywords Earthquake simulation - Soil structure interaction - Bridge structure - High performance computing

\section{Introduction}

The seismic demands of a bridge structure subjected to a particular ground motion can be estimated through an equivalent analysis of a mathematical model that incorporates the behavior of the superstructure, piers, footing and soil system. The idealized model should properly represent the actual geometry, boundary, conditions, and gravity load distribution [1-3]. Nonlinear modeling and analysis allows more accurate determination of stresses, strains, deformations and forces of critical components results than can be used for the final design of the bridge system. 
This project studies the effects of specially input excitation at an existing bridge site. A nonlinear finite element of this bridge site including the bridge structure, pile group and supporting foundation soil, is developed and 2D plane strain conditions. The computational model is analyzed using OpenSees, a software platform developed at the Pacific Earthquake Engineering Research (PEER) Center [4]. Carefully calibrated nonlinear stress-strain models are employed for both bridge and soil materials, in order to realistically reproduce actual site conditions. Seismic input motions are defined as equivalent excitation forces. In this study, the input excitation is prescribed at different edges of incidence at longitudinal planes to examine the significance of spatially varying excitation on the overall response of the bridge system.

Model based numerical simulation of seismically induced site effects commonly assumes a uniform base excitation. This assumption may not be realistic for structures whose horizontal dimensions are comparable to the wave length of foundation ground motions. In this presentation, some preliminary results of a computational study on the effects of spatially varying input excitation at an existing bridge site were studied. The bridge is located near Karad on Krishna river.

1.1 Dynamic analysis methods for seismic bridge design

\subsubsection{Analysis methods used for seismic bridge design}

- The single-mode method (single-mode spectral and uniform-load analysis) assumes that seismic load can be considered as an equivalent static horizontal force applied to an individual frame in either the longitudinal or transverse direction. The equivalent static force is based on the natural period of a single degree of freedom (SDOF) and code-specified response spectra. This single-mode method (sometimes referred to as equivalent static analysis) is best suited for structures with well-balanced spans with equally distributed stiffness.

- Multimode spectral analysis assumes that member forces, moments, and displacements due to seismic load can be estimated by combining the responses of individual modes using methods such as the complete quadratic combination (CQC) method and the square root of the sum of the squares (SRSS) method. The CQC method is adequate for most bridge systems and the SRSS method is best suited for combining responses of well-separated modes.

- The multiple support response spectrum method provides response spectra and the peak displacements at individual support degrees of freedom by accurately accounting for the spatial variability of ground motions, including the effects of incoherence, wave passage, and spatially varying site response. This method can be used for multiply supported long structures.

- The time-history method is a numerical step-by-step integration of equations of motion. It is usually required for critical/important or geometrically complex bridges. Inelastic analysis provides a more realistic measure of structural behavior when compared with an elastic analysis.

\section{Site description}

There is an existing bridge on Guhagar-Karad-Jat-Vijapur, S.H. 78 at 141/200 across Krishna River near Karad. Existing bridge is constructed in between year 1936 and 1939 and has 14 spans of about $19.50 \mathrm{~m} \mathrm{c} / \mathrm{c}$ with $7.30 \mathrm{~m}$ roadway. Approximate height of existing bridge from bed of river is about 15-16 m. Existing bridge is masonry arch with spandrel high level submersible type.

The new bridge proposal is prepared as high level bridge by keeping pier positions in line with the piers of existing bridge. Hence proposed span arrangement is 39.5, 38.0, $40.0,40.0,40.0,40.0,39.0$ and $39.0 \mathrm{~m}$. The total length of the bridge is $315.50 \mathrm{~m}$.

The bridge is having two lane with $7.5 \mathrm{~m}$ clear road width between the kerbs and footpath $1.5 \mathrm{~m}$ width on $\mathrm{D} / \mathrm{S}$. 8-span composite structures with pre-stressed concrete box and RCC piers to provide continuity. Piers are supported on six pile groups, each of which is RCC piles. The foundation data is composed of mainly medium to dense clayey sand. In addition, fractured amygdaloidal basalt layers are present. The hard rock is located at 23-25 m depth.

The Karad bridge near Pune, India, was selected as development of a performance-based earthquake engineering design and assessment methodology for earthquake simulations. A 2D, advanced nonlinear computational model of the structure-foundation-soil system has been developed using Gid and OpenSees for earthquake simulations, the PEER software framework for seismic response simulation of structural and geotechnical systems (Fig 1). In this paper a simplified, yet improved definition of the seismic excitation along the boundaries of the soil domain included in the finite element model of the structure-foundation-soil system. This seismic excitation assumes vertically incident shear waves and a linear elastic, undamped, and homogeneous semiinfinite half-space underlying the nonlinear soil region modeled. It is also consistent with free field (rock and soil) actual earthquake records representative of the seismicity of Karad bridge. The proposed treatment of the boundary conditions includes transmitting/absorbing boundaries in order to limit the occurrence of spurious seismic wave 
Fig. 12 D modelling using Gid of existing Karad bridge

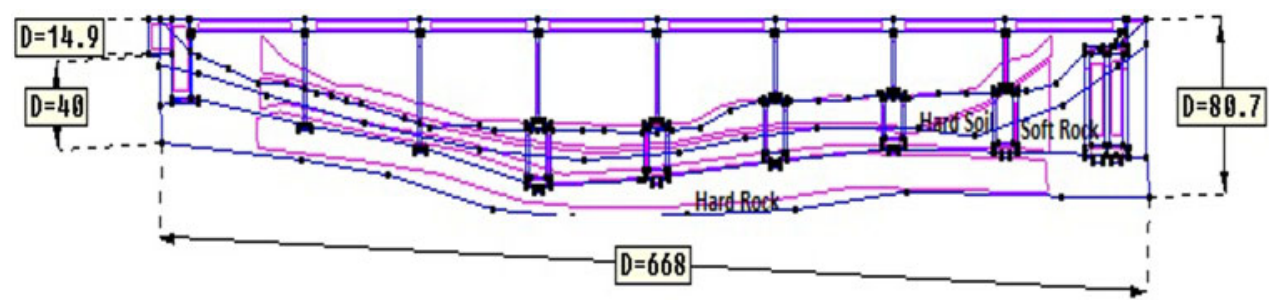

reflections along the boundaries of the modeled soil medium. Using simple (single wavelet) and complex (earthquakelike) incident wave motions, the dynamic response of the bridge-foundation-soil system obtained using the above modeling [5,6]. The computational model developed is exercised to assess the effects of soil nonlinearity (including lateral spreading) on the seismic response of the structurefoundation-soil system.

\subsection{Equation of motion}

The response of a structure depends on its mass, stiffness, damping, and applied load or displacement. The structure could be excited by applying an external force $p(t)$ on its mass or by a ground motion $u(t)$ at its supports. Since the seismic loading is induced by exciting the support, we focus mainly on the equations of motion of an SDOF system subjected to ground excitation (Fig. 2).

The displacement of the ground motion $u_{g}$, the total displacement of the single mass $u_{t}$, and the relative displacement between the mass and ground $u$ (Fig. 3) are related by Eq. (1)

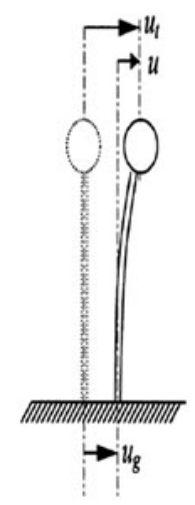

Fig. 2 Earthquake-induced motion of an SDOF system $u_{t}=u+u_{g}$

By applying Newton's law and D'Alembert's principle of dynamic equilibrium, it can be shown that

$f_{1}+f_{d}+f_{s}=0$

where $f_{1}$ is the inertial force of the single mass and is related to the acceleration of the mass by $f_{I}=m \ddot{u}_{t} ; f_{d}$ is the damping force on the mass and related to the velocity across the viscous damper by $f_{d}=c \dot{u} ; f_{s}$ is the elastic force exerted on the mass and related to the relative displacement between the mass and the ground by $f_{s}=k u$, where $\mathrm{k}$ is the spring constant; $\mathrm{c}$ is the damping ratio; and $\mathrm{m}$ is the mass of the dynamic system.

Substituting these expressions for $f_{I}, f_{d}$, and $f_{s}$ into Eq. (2) gives

$m \ddot{u}_{t}+c \dot{u}+k u=0$.

The equation of motion for an SDOF system subjected to a ground motion can then be obtained by substituting Eq. (1) into Eq. (3), and is given by

$m \ddot{u}+c \dot{u}+k u=-m \ddot{u}_{g}$.

\subsection{Modeling of bridge structure}

Various assumptions made for analytical modeling of bridge structure are

1. The structure is assumed to be symmetric and idealized as $2 \mathrm{D}$ structure.

2. The structure is assumed to be simply supported for analysis but modeled as whole bridge structure.

3. The super structure remains elastic during the earthquake excitation.

4. Piles are end bearing piles.

5. Structure excited by earthquake ground motion widthwise and lengthwise i.e. 2 way earthquake application.

6. Abutment-pier interaction and pier-pier interaction is not considered.
Fig. 3 2D meshing of existing Karad bridge

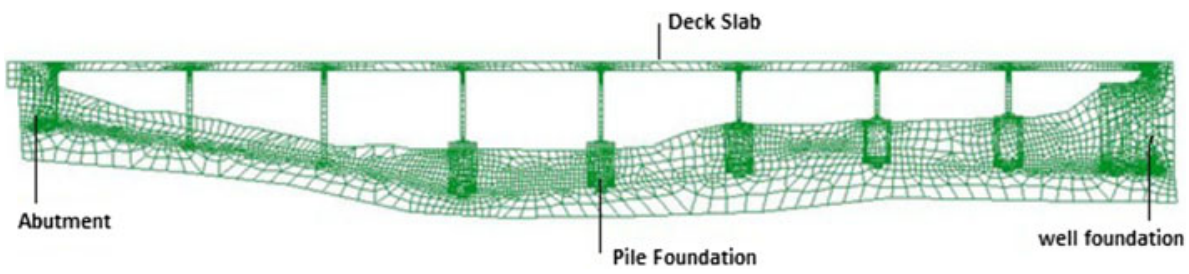


7. The force deformation behaviour of the bearing system is linear with viscous damping.

\section{Finite element modeling}

A 2D nonlinear model of the Karad bridge, including the structural components as substructure, superstructure, piers, and supporting piles was developed in Gid (pre-post processing software). The material exists as ground strata were medium sand at the top layer, fractured amygdaloidal basalt as middle layer and hard rock (compact basalt) at the bottom layer. The RCC substructure consists of pile foundation are reinforced concrete structure with M40 grade of concrete. The superstructure consists of reinforced concrete bridge piers (M40 grade), prestressed concrete deck slab and PTFE bearings. The entire 2D modeling and meshing was done using element quad PlaneStrain2D quadrilateral elements in OpenSees [4, 7-9]. The pile groups supporting the bridge piers are first lumped in the bridge transversal direction and then modeled using 2D nonlinear element (Fig. 3). The prestressed concrete structure modeled as co-rotational truss element and Steel02 material which defined the initial stresses developed. The bearings provide the connection between the super structure and sub structure of the bridge. To model the bearing zero-length element and the hysteretic material was used. Damping effect was provided with specifying the corresponding nodes. The soil domain is analyzed under the assumption of plane strain condition. The materials in the various layers of the soil domain are modeled using quadrilateral element. The lateral and base boundary conditions for the computational soil domain are modeled using a zero length elements for transmitting/absorbing earthquake forces.

3D model includes nonlinear fiber beam-column elements (for bridge piers and foundation piles). The material properties of various bridge components remain same as that of 2D model. 20 node solid elements were used to define the structural component with structured meshing $[10,11]$.

\subsection{Methodology for simulations}

As stated above, the seismic input is defined in terms of equivalent nodal forces (or effective earthquake forces), which are proportional to the acceleration of the incident seismic wave, applied in the horizontal direction along the base of the soil domain included in the finite element model. Three sets of scaled actual earthquake ground motions Koyana, Bhuj and Tabas corresponding to three different hazard levels $(50,10$, and $2 \%$ in 50 years, respectively) were used. Amongst these three earthquakes, since the bridge is located near the Koyana earthquake epicenter, the site conditions are seismically and geologically similar. The TCL scripting incorporated with nodes, elements and boundary conditions, material properties,

Table 1 Stress comparison with different analysis methods

\begin{tabular}{llll}
\hline Analysis method & Maximum & Maximum \\
stress $\left(\mathrm{N} / \mathrm{m}^{2}\right)$ & $\begin{array}{l}\text { deformation } \\
(\mathrm{m})\end{array}$ \\
\hline $\begin{array}{c}\text { Analysis of bridge as } \\
\text { whole structure }\end{array}$ & $3.67 \mathrm{E}+08$ & 0.091 \\
$\begin{array}{c}\text { Analysis of bridge by } \\
\text { convention method } \\
\text { taking single pier }\end{array}$ & $\begin{array}{c}\text { Both span on } \\
\text { condition } \\
\text { One span off } \\
\text { condition }\end{array}$ & $3.64 \mathrm{E}+06$ & 0.0174 \\
\hline
\end{tabular}

Table 2 Deformation of middle span

\begin{tabular}{llll}
\hline Mode & $\begin{array}{l}\text { Frequency } \\
(\mathrm{Hz})\end{array}$ & $\begin{array}{l}\text { Time period } \\
(\mathrm{s})\end{array}$ & $\begin{array}{l}\text { Deformation middle span } \\
(\mathrm{mm})\end{array}$ \\
\hline 1 & 0.737 & 8.53 & 0.48 \\
2 & 0.87 & 7.22 & 0.5 \\
3 & 1.07 & 5.87 & 0.5 \\
4 & 1.27 & 4.95 & 0.49 \\
5 & 1.494 & 4.21 & 0.47 \\
6 & 1.57 & 4.00 & 0.32 \\
\hline
\end{tabular}

Table 3 Modal analysis results of the pier

\begin{tabular}{llll}
\hline Mode & Frequency $(\mathrm{Hz})$ & Time period $(\mathrm{s})$ & Deformation $(\mathrm{cm})$ \\
\hline 1 & 1.269 & 4.95 & 2.2 \\
2 & 1.804 & 3.48 & 1.58 \\
3 & 2.811 & 2.24 & 1.43 \\
4 & 4.141 & 1.52 & 2.6 \\
5 & 4.454 & 1.41 & 2.14 \\
6 & 10.709 & 0.59 & 2.2 \\
\hline
\end{tabular}

Table 4 Simulation results on Param Yuva

\begin{tabular}{lrlcl}
\hline $\begin{array}{l}\text { Sr. } \\
\text { No. }\end{array}$ & $\begin{array}{l}\text { No. of } \\
\text { nodes }\end{array}$ & $\begin{array}{l}\text { No. of } \\
\text { elements }\end{array}$ & $\begin{array}{l}\text { Simulation } \\
\text { time }(\mathrm{s})\end{array}$ & $\begin{array}{l}\text { Earthquake } \\
\text { Applied }\end{array}$ \\
\hline 1. & 9,778 & 11,314 & 7,880 & Tabas \\
2. & 12,668 & 15,478 & 9,900 & Tabas \\
3. & 15,669 & 17,880 & 15,890 & Tabas \\
4. & 8,999 & 12,566 & 9,910 & Bhuj \\
5. & 10,550 & 13,899 & 15,860 & Bhuj \\
6. & 13,556 & 17,886 & 18,790 & Bhuj \\
7. & 9,778 & 11,314 & 6,980 & Koyana \\
8. & 12,668 & 15,478 & 9,910 & Koyana \\
9. & 15,669 & 17,880 & 10,160 & Koyana \\
\hline
\end{tabular}




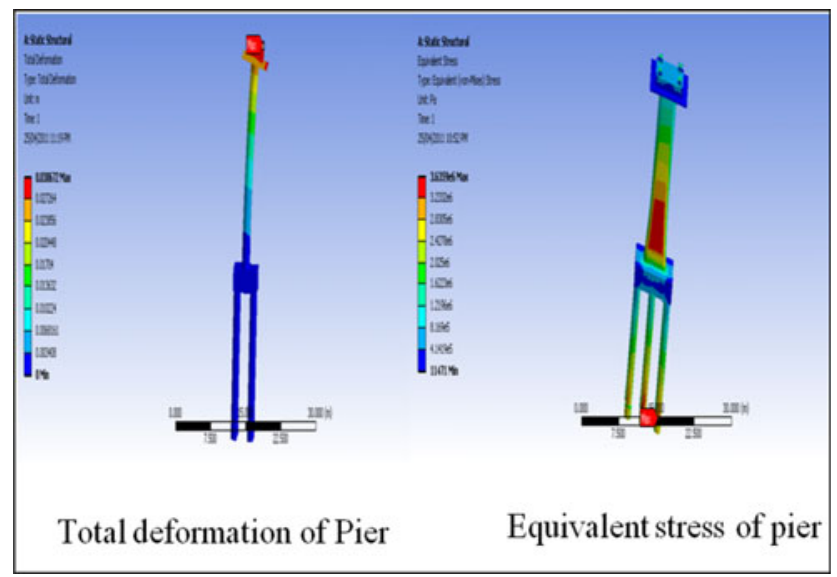

Fig. 4 Deformation and stress of middle pier

simulations methodology transient analysis parameters. The analysis involves, integrator new mark 0.5, 0.25, constraints transformation 1.0, algorithm linear, numberer plain, system profile SPD.

Fig. 5 Stress variation of pier

\subsection{Static analysis of bridge}

Table 1 shows the total deformation and equivalent stresses of the single middle pier. For analysis of bridge as whole structure the value of maximum stress and maximum deformation corresponds to bridge middle span of bridge.

\subsection{Modal analysis of bridge middle}

Table 2 shows the values for deformations of bridge middle span in modal analysis. The modal analysis results are of Bhuj earthquake, giving maximum deformation of 0.5 $\mathrm{mm}$ for $2 \mathrm{nd}$ and $3 \mathrm{rd}$ mode of earthquake simulation.

\section{Analysis results}

Transient analysis of a bridge structure is carried out by applying three different ground motions. The results were plotted for the middle pier (Fig. 4), where it is observed that the stress values and deformation values are maximum.
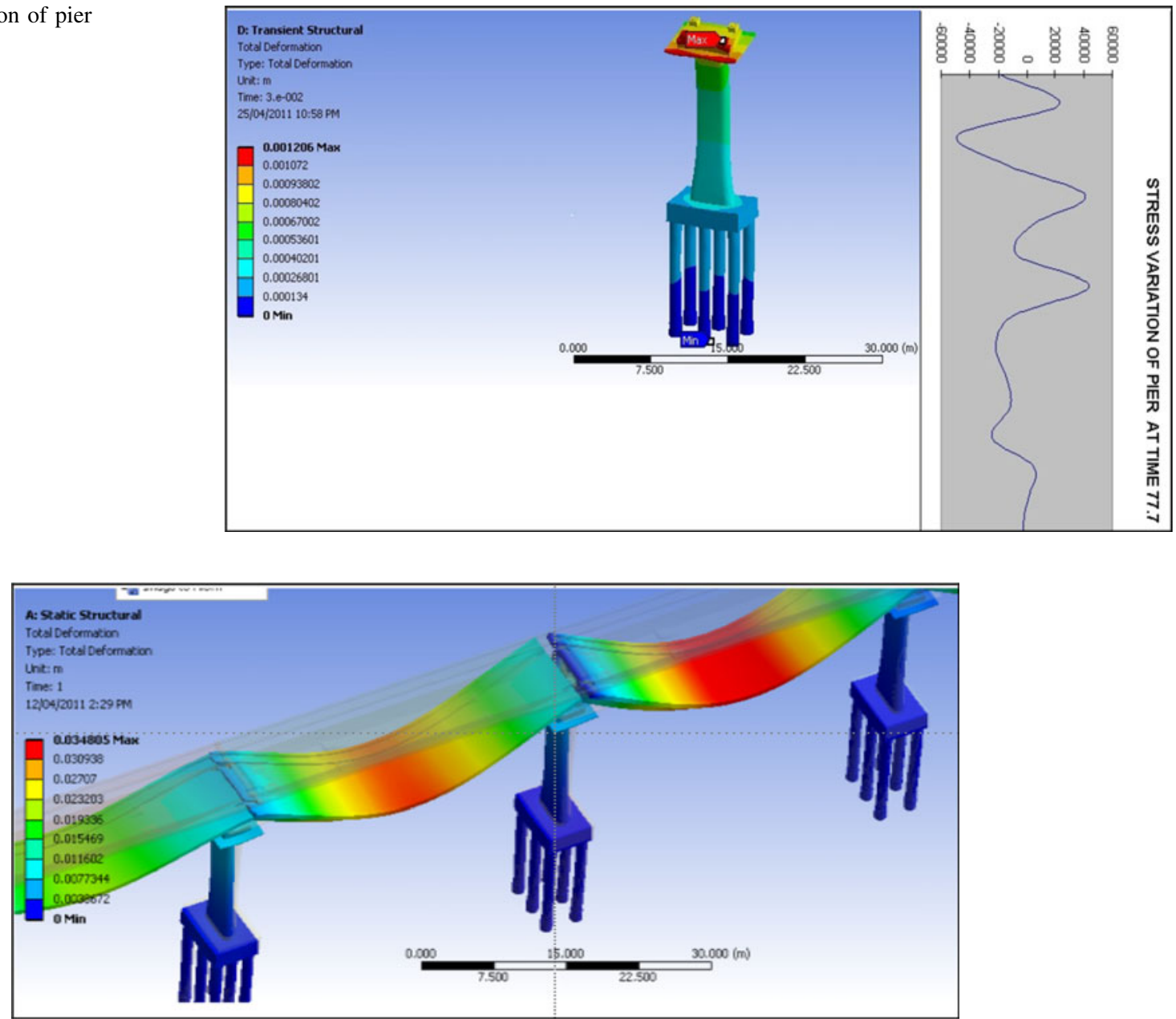

Fig. 6 Deformations for the middle span 
The response of the bridge structure is nonlinear. The stress distribution of the middle pier is shown below in Fig. 5 . The modal analysis results for deformations are given in Table 3, shows maximum deformation of $2.6 \mathrm{~cm}$ for the middle pier structure.

To illustrate the procedure for defining the seismic input, a rectangular, homogeneous, undamped, elastic soil domain ( 1,000 by $40 \mathrm{~m}$ in size) under plane stain condition is discretized into the finite element model shown in Fig. 2. Zero length elements are developed in the model and boundary conditions are given as per the actual bridge. Bridge bearings have given the fixation and freeness as per the site conditions. Earthquake force application was based on time History analysis. The TCL scripts for various earthquakes loading was formulated and results obtained was given in Table 4.

Above results shows a linear progress as the intensity of earthquake rises and as the element sizes increase in numbers. The iterations used for earthquake are 20,000 incremental dynamic iterations for each earthquake on 32 no. of processors.

\section{Conclusion}

1. Results shown that the analysis method may play a major role in stresses of the bridge members.

2. It is seen that $3 \mathrm{D}$ analysis of the bridge structure gives higher stress values than conventional design procedure [12].

3. Transient analysis of the bridge structure gives higher stress values than conventional design procedure for seismic analysis. It is felt that the structure should be analyzed with time history, as the maximum deformation value we get from this study is $247 \mathrm{~mm}$, which may induce longitudinal/transversal displacement of pier.

4. Soil-structure interaction may play a major role in the seismic response of a bridge structure. Specifically, soil layers of low stiffness and strength may result in permanent displacement of the abutments and foundations, thus imposing important kinematics conditions to the bridge structure. A study to illustrate such phenomena is undertaken based on $2 \mathrm{D}$ and $3 \mathrm{D}$ nonlinear dynamic finite-element (FE) modeling and analysis (for Karad bridge configuration under a three seismic excitation). The FE model and nonlinear solution strategy are built in OpenSees. Gid was employed for the mesh generation in pre-processing. Based on the simulation results, the overall seismic response behavior is examined with local deformations/stresses at selected critical locations. It is shown from Fig. 6 that permanent ground deformation induced settlement and longitudinal/transversal displacements of the spans of the bridge, abutments and deep foundations. The relatively massive approach ramps may also contribute to this simulated damage condition, which imposes large stresses on the bridge foundations, supporting piers, and superstructure.

5. The effect of linear elastic versus elastoplastic soil constitutive behavior on the propagation of vertically incident shear waves in the computational domain is examined. It is found that soil nonlinearity has a significant effect on the wave propagation through the foundation soil.

\section{References}

1. Elgamal A, Yang Z, Parra E, Ragheb A (2003) Modeling of cyclic mobility in saturated cohesionless soils. Int J Plast 19(6):883-905

2. Kammerer A et al (2000) Cyclic simple shear testing of Nevada sand for PEER center project 2051999. Research Report No. UCB/GT/00-02, University of California at Berkeley, Berkeley

3. Yang Z, Elgamal A, Parra E (2003) A computational model for liquefaction and associated shear deformation. J Geotech Geoenviron Eng 129:1119-1127

4. McKenna F, Fenves GL (2001) The OpenSees command language manual, Version 1.2. Pacific Earthquake Engineering Research Center, University of California at Berkeley, Berkeley. http://opensees.berkeley.edu/. Accessed 10 July 2013

5. Zhang Y, Yang Z, Bielak J, Conte JP, Elgamal A (2003) Treatment of input excitation and boundary conditions in nonlinear seismic analysis of a bridge ground system. In: Proceedings, 16th ASCE Engineering Mechanics conference, University of Washington, Seattle, July 16-18, CD-ROM

6. Conte JP, Elgamal A, Yang Z, Zhang Y, Acero G, Seible F (2002) Nonlinear seismic analysis of a bridge ground system. In: Proceedings of the 15th ASCE Engineering Mechanics conference, Columbia University, New York, June 2-5, CD-ROM

7. Cornell CA, Krawinkler H (2000) Progress and challenges in seismic performance assessment. PEER Center News, 3(2), Spring 2000

8. Kramer SL (1996) Geotechnical earthquake engineering. Prentice Hall, Upper Saddle River

9. Lysmer J, Kuhlemeyer RL (1969) Finite dynamic model for infinite media. J Eng Mech Div ASCE 95(EM4):859-877

10. Yoshimura C, Bielak J, Hisada Y, Fernández A (2003) Domain reduction method for three-dimensional earthquake modeling in localized regions, part II: verification and applications. Bull Seismol Soc Am 93(2):825-840

11. Schnabel PB, Lysmer J, Seed HB (1972) SHAKE: A computer program for earthquake response analysis of horizontally layered sites. Report No. EERC 72-12, Earthquake Engineering Research Center, University of California at Berkeley, Berkeley

12. Somerville P, Collins N (2002) Ground motion time histories for the Humboldt bay bridge. Report of the PEER performance based earthquake engineering methodology testbed program, Pacific Earthquake Engineering Research Center, University of California at Berkeley, Berkeley. http://peer.berkeley.edu/. Accessed 10 July 2013 